\title{
INTERNATIONAL COMMITTEE ON BACTERIOLOGICAL NOMENCLATURE
}

\author{
Members and Officers- 1952
}

\begin{abstract}
Provision 4 of the International Bacteriological Code of Nomenclature established a permanent International Committee on Bacteriological Nomenclature "so constituted that wherever practicable each country is represented by at least one member and no country by more than five. Nominations for membership are made preferably by one or more of the microbiological societies in each country." If nominations are not so made, they may be submitted by any member of the International Committee to one of the Permanent Secretaries. Acceptance will be indicated by the Permanent Secretaries, and ad interim membershipgiven pending act on of the International Committee and the next International Congress of the International Association of Microbiologists.
\end{abstract}

A list of the officers and members of the International Committee on Bacteriological Nomenclature as approved at the $F$ ifth International Congress of the International As sociation held at Rio de Janeiro, Brazil, in August 1950 was printed on the cover pages of each issue of the BULLETIN during the year 1951. That there were inaccuracies in the list was recognized. Corrections have been made insofar as they have come to light. Following is a list of the members of the delegations from the several countries as shown by the records of the Permanent Secretaries. Some addresses are unknown. The Permanent Secretaries will be grateful for information relative to any inaccuracies.

\section{OF FICE}

KLUYVER, Prof. A. J. - Technical High School, Nieuwe - Laan 5, Delft, Holland.

PREVOT, Dr. A. R. - Institut Pasteur, 25 Rue du Dr. Roux, Paris XVe. France.

Permanent Secretary for Medical and Veterinary Bacteriology

COWAN, Dr. S. T. - National Collection of Type Cultures, Central

Public Health Laboratory, Colindale Avenue, London N. W. 9

England.

Permanent Secretary for Non-Medical Bacteriology

WIKEN, Prof. Torsten. - Inst. f. Bakteriologie u. Gärungsbiologie.,

Eidgenoss. Techn. Hochschule, Universitătstrasse 2, Zärich 6, Switzerland. 


\section{MEMBERS OF COMMITTEE}

\section{ARGENTINA}

SORDELli, Prof. A. - Posodas 1553, Martinez F.C. N. G. B. N., Buenos Aires.

SORIANO, Prof. Ing. Santos - Instituto de Microbiologia Agricola, Villa Orturar-Suc. 17, Buenos Aires.

\section{AUSTRALIA}

MUNCH-PETERSON, Dr. E. - Div. Animal Health and Nutrition, Flemington Road and Park Street, Parkville, N. 2, Victoria.

RUBBO, Prof. S. D. - Department of Bacteriology, The University, Melbourne, Victoria.

SkERMAN, Prof. V. B. D. - Dept. Bacteriology, Medical School, Queensland University, Herston Road, Brisbane, Queensland.

\section{AUSTRIA}

CHIARI, Prof. Hermann - Vorstand Pathologisch-Anatomisches Institut, Universitat, Franzenring 6, Vienna 9.

EISLER - TERRAMARE, Prof. Michael - Staatl. Serotherapeut. Institut, Universität, Vienna 10.

JANKE, Prof. Alexander - Technische Hochschule, Waldeckgasse 3, Wien XVIII 110.

KRENN, Prof. Ing. J. - Lehrkanzel landwirtschaft. Mikrobiol., Gregor Mendelstr. 33, Vienna. 18.

SZYLVINI, Prof. Ing. - Institut fur angewandte Mikrobiologie, Hochschule für Bodenkultur, Michaelstr. 25, Vienna 18.

\section{BELGIUM}

BRUYNOGHE, Prof. Richard - Department of Bacteriology, University of Louvain.

RENAUX, Prof. Ernest - Faculté de Médecine et de Pharmacie, Université Libre de Bruxelles, 115 Boulevard de Waterloo, Brussels.

\section{BR AZIL}

BIt ANCOURT, Dr. Agesilau - Instituto Biologico, Caixa Postal 7119 Săo Paulo, Estado de São Paulo.

CRUZ, Dr. Oswaldo, filho - Instituto Oswaldo Cruz, Caixa Postal 926, Rio de Janeiro, D. F.

DA ROCHA LAGOA, Dr. Francisco de Paulo - Instituto Oswaldo Cruz, Caixa Postal 926 Rio de Janeiro, D. F.

PENHA, Dr. Adolpho Martins - Instituto Biologico, Caixa Postal 7119 Sao Paulo (Estado de Sao Paulo).

PENIDO, Dr. J. C. N. - Instituto Oswaldo Cruz, Caixa Postal 926, Rio de Janeiro D. F. 
BACTERIOLOGICAL NOMENCLATURE AND TAXONOMY

\section{BRITISH WEST INDIES}

THAYSEN, Dr. A.C. - Director, Colonial Microbiological Institute, P. O. Box 584, Trinidad.

\section{BULGARIA}

MARKOFF, Prof. W. - Dept. of Bacteriology, Univ. of Sofia, 3 Rue Dounau, Sofia.

PETROFF, Prof. T. - Department of Hygiene, University of Sofia, Sofia.

\section{CANADA}

LOCHHEAD, Dr. A. G. - Division of Bacteriology, Central Experimental Farm, Ottawa, Ontario.

MORIN, Prof. E. J. - Dept. Bacteriology, Laval University, Quebec, P.Q.

MURRAY, Prof. E.G.D. - Dept. Bacteriology, McGill University, Montreal P. Quebec.

REED, Prof. Guilford B. - Dept. Bacteriology, Medical School, Queens University, Kingston, Ontario.

VAN ROOYEN, Dr. C.E - Connaught Laboratories, University of Toronto, Toronto 5, Ontario.

\section{CHILI}

GRAF-MARIN, Dr. Alberto - Professor of AgricuItural Microbiology, Universidad de Chile, Casilla 370, Santiago, Chile.

SAN MIGUEL, Dr. Julio - Faculdad de Ciencias Pecuarias y Medicina Veterinaria, Universidad de Chile, Casilla 370, Santiago.

VACCARRO, Dr. Hugo - Instituto de Microbiologia e Imunologia, Casilla 370 , Santiago.

WILHELM, Dr. Ottmat - Prof., Universidad de Concepcion, Concepcion

\section{CZECHOSLOVAKI A}

MALEK, Dr. Ivan. - Department of Bacteriology, University of Praha, Preslova 7, Praha.

RASKA, Dr. Karel - State Institute of Public Health, Srobárova 48, Praha III.

\section{DENMARK}

BUCHWALD, Prof. Dr. N. Fabritius - Kgl. Veterinariog Landbohjskole, Bülowsvej, Copenhagen.

CHRISTIANSEN, Prof. M. - Serum Lab.; Veterinary School, Bülowsvej, Copenhagen.

KAUFFMANN, Dr. F. - Statens Seruminstitut, Amager Blvd. 80, Copenhagen S.

ØRSKOV, Dr. J. - Statens Seruminstitut, Amager Blvd. 80, Copenhagen S. 
EIRE

GRIMES, Prof. Michael - Dairy Institute, University of Cork, Cork.

FINLAND

VARTIOVAARA, Dr. Unto - Dept. of Microbiology, The University, Helsinki.

FR ANCE

Hauduroy, Prof. Paul - Directeur du Centre de Collection de Types Microbiens, 19 Ave. César Roux, Lausanne, Switzerland.

LEPINE, Dr. P. - Institut Pasteur, 25 Rue du Dr. Roux, Paris XVe. LWOFF, Dr. A. - Institut Pasteur, 28 Rue du Dr. Roux, Paris XVe.

PREVOT, Dr. A. R. - Institut Pasteur, 25 Rue de Dr. Roux, Paris XVe. SEgRetaIN, Dr. G. - Institut Pasteur, 25 Rue du Dr. Roux, Paris XVe.

\section{GERMANY}

BOECKER, Prof. Dr. Eduard - Direktor Robert Koch Institut, Föhrerstrasse 2, Berlin 65.

HAUPT, Prof. Dr. Herbert - Veterinärhygienisches Institut, Frankfur terstrasse 87, Giessen.

MÜller, Prof. Dr. Reiner - Direktor, Hygienisches Institut, Fürst Pücklerstr., 56, Köln-Lindenthal.

SEELEMANN, Prof. Dr. M. - Institut für Milchhygiene, Hermann Weigmannstr $3 / 11 \mathrm{Kiel}$.

STAPP, Prof. Dr. C. - Direktor Biologische Zentralanstalt, 20b Braunschweig-Gliesmarode. Messeweg 25.

\section{GREAT BRITAIN AND NOR THERN IRELAND}

ANDREWES, Dr. Christopher H. - National Institute for Medical Re search, The Ridgeway, Mill Hill, London N. W. 7.

MILES, Dr. A. A. - National Institute for Medical Research, The Ridgeway, Mill Hill, London N. W. 7.

RAMSBOTTOM, Dr. J. - British Museum (Natural History, ) Cromwell Road, London S. W. 7.

WILsON, Dr. G.S. - Public Health Laboratory Service, 38 Old Queen Street, Westminster, London S. W. 1.

\section{HUNGAR Y}

MANNINGER, Prof. R. - Director, State Veterinary Hygienic Institute, Hungăria Korut 23-26, Budapest XIV.

\section{ISRAEL}

ASCHNER, Dr. M. - Dept. Bacteriology, Hebrew University, Jerusalem 
IT ALY

ARNAUDI, Prof. Carlo - Instituto di Microbiologia della Facoltà di Agraria, Universita di Milano, Via Celaria, Milan.

CIFERRI, Prof. R. - Instituto Botanico, Università di Pavia, Via Scopoli, P.O. Box 165, Pavia.

DE ROSSI, Prof. Gino. - Via Buccari 3, Rome.

PENSO, Dr. G. - Instituto Superiore di Sanita, Viale Regina Margherita 299, Rome.

PUNTONI, Prof. V. - Instituto d'Igiene, Università Roma, Rome.

JAPAN

ASAI, Prof. Dr. Toshinobu - Faculty of Agriculture, Univ. of Tokyo, Mukogaoka-Yayoimachi, Bunkyo-ku, Tokyo.

KOJIMA, Dr. Sabura - Director of the National Institute of Health, 1 , Shiba-Shirokane-Daimachi, Minato-ku, Tokyo.

KOMINAMI, Dr. Kiyoshi - Nagao Research Institute, 387, 6, Cho-me, Kitashinagawa, Shinagawa-ku, Tokyo.

\section{NETHERLANDS}

JULIUS, Prof. Dr. H. W. - Laboratorium voor Hygiene, Univ. Utrecht, Catharijnesingel 59, Utrecht.

KLUYVER, Prof. Dr. A. J. - Technical High School, N euwe Laan 3, Delft.

\section{NEW ZEALAND}

BUDDLE, M. B., (B. V.Sc.) - Wallaceville Animal Research Station, Private Bag. Wellington.

KIRSTNER, Dr. L. - Medical School, University of Otago, Dunedin, Cl. WHITEHEAD, Dr. H. R. - Dairy Research Institute, Massey College, Palmerston North.

NOR W AY

HENR IKSEN, Dr. S. Dick - Statens Institutt for Folkehelse, Geitmyrsvegen 75 , Oslo.

THJळт TA, Prof. Th. - Bacteriological Institute, University of Oslo, Oslo.

VOSS, Prof. J. - State Institutt for Folkehelse, Geitmeyrsvegen 75 , Oslo.

\section{POLAND}

LEGEZYNSKI, Prof. St. - Department of Bacteriology, State Institute of Hygiene, Chocimska 28, Warsaw .

PRZESMYCKI, Prof. F. - State Institute of Hygiene, Chocimska 28, Warsaw.

SZYMANOSKI, Prof. Dr. F. - Dept. of Bacteriology, The University, Lodz, Poland. 


\section{RUMANIA}

IONESCU-MIHAIESTI, Prof. Dr. C. - Faculty of Medicine, Splaiul Dr. Carol Davilla 6, Bucharest VI.

ZOTTA, Prof. Dr. G. - Splaiul Dr. Carol Davilla 6, Bucharest VI.

\section{SPAIN}

FALCO, Dr. A. Ruiz - Instituto Nacional de Hygiene, Madrid.

\section{SWEDEN}

GARD, Prof. Sven - State Bacteriological Laboratory, Karolinska Ins titutet, Box 764, Stockholm 1.

HÜLPHERS, Prof. Gustav - Dept. Bacteriology, Veterinary College, Stockholm.

NILSSON, Prof. Ragner - Kung1. Lantbrukshogskölan, Uppsala.

\section{SWITZERLAND}

DOERR, Prof. R. - Hygienisches-Institut der Universität, Basel. FREI, Prof. W. - Veterinär-Pathologisches Institut, University of Zürich, Zürich.

\section{UNITED STATES OF AMERICA}

ALLEN, Dr. Oscar N. - Department of Bacteriology, University of Wisconsin, Madison 6, Wisconsin.

BROWN, Dr. J. Howard - 1640 Gorsuch Avenue, Baltimore 18, Maryland.

BUCHANAN, Prof. R. E. - Room 24 Curtiss Hall, Iowa State College, Ames, Iowa.

VAN NIEL, Prof. C.B. - Hopkins Marine Station, Pacific Grove, California.

\section{URUGUAY}

HORMAECHE, Prof. E.- Instituto de Higiene, Faculdad de Medicina, Av. A. Ricaldoni, 3051, Montevideo.

$$
\text { U.S.S.R. }
$$

HOROWITZ-WLASSOWA, Prof. - 1 Linia, 54, App. 3, Leningrad 53, W. O.

ISSATCHENKO, Prof. Dr. B. - Institute of Experimental Medicine, 96 Baltiiskii poselok 13, Moscow 96.

\section{LIFE MEMBERS}

ST. JOHN-BROOKS, Dr. Ralph - Department of Bacteriology, Trinity College, Dublin, Ir eland.

BREED, Prof. R.S. - 6 Sunset Drive, Geneva, New York, U.S. A. 